\title{
An analogue circuit to study the forced and quadratically damped Duffing oscillator
}

\section{Udayprakash Raghunath Singh}

Department of Electronics and Communication Engineering,

Sir Padampat Singhania University,

Bhatewar, Udaipur 313-601, Rajasthan, India

Fax: +91-2957-226094

Email: udayprakash.singh@spsu.ac.in

\section{Ghanshyam Purohit*, Anjali Sharma and Vinod Patidar}

Department of Physics,

Sir Padampat Singhania University,

Bhatewar, Udaipur 313-601, Rajasthan, India

Fax: +91-2957-226094

Email: ghanshyam.purohit@spsu.ac.in

Email: anjalishar.2008@gmail.com

Email: vinod.patidar@spsu.ac.in

*Corresponding author

\begin{abstract}
In this communication we study the forced and quadratically damped Duffing oscillator system experimentally through an analogue circuit. For this purpose we design a circuit and observe its dynamical behaviour under various sets of control parameters and also compare the experimental results with the numerical simulation results.
\end{abstract}

Keywords: nonlinear damping; Duffing oscillator; chaos; periodic attractor.

Reference to this paper should be made as follows: Singh, U.R., Purohit, G., Sharma, A. and Patidar, V. (2017) 'An analogue circuit to study the forced and quadratically damped Duffing oscillator', Int. J. Nonlinear Dynamics and Control, Vol. 1, No. 1, pp.87-96.

Biographical notes: Udayprakash Raghunath Singh is working as an Associate Professor of Electronics and Communication Engineering and Controller of Examination at Sir Padampat Singhania University, Udaipur, India. He has obtained his BE (Industrial Electronics) degree from Nagpur University, Nagpur, India in 2000 and MTech (VLSI Design) degree from MITS Lakshmangarh, Sikar, Rajasthan, India in 2009. He has about 15 years of teaching and research experience. He is working in the field of nonlinear dynamics and circuits.

Ghanshyam Purohit is working as a Professor of Physics and Associate Dean Research at Sir Padampat Singhania University, Udaipur, India. He has obtained his $\mathrm{PhD}$ in Theoretical Atomic Physics from M.L.S. University, Udaipur in 2005. He has over 40 research publications in the field of atomic physics, nonlinear dynamics and chaos. He has visited ICTP, Trieste (Italy), 
Max Planck Institute for Nuclear Sciences, Heidelberg (Germany), ITAMP, Harvard University, Cambridge (USA) as a Visiting Scientist, besides this, he has presented research work at various international conferences held in UK, USA, Japan, Germany, France, Austria, Italy, Sweden, Switzerland, Ireland, China, etc.

Anjali Sharma obtained her $\mathrm{PhD}$ degree on the 'study of dynamical behaviour of some of the ubiquitous nonlinear oscillators under nonlinear damping' from Sir Padampat Singhania University (SPSU), Udaipur, Rajasthan, India in 2015. Prior to this, she completed her BSc degree from C.C.S. University, Meerut, UP, India in 2007 and MSc (Physics) degree from Banasthali University, Rajasthan, India in 2009. She then worked as a Research Fellow in a DST, Government of India sponsored project during 2010-2012 at SPSU. She also worked as an Assistant Professor of Physics in B.N.P.G. College, Udaipur and Mewar Univesity, Chittorgarh, during 2012-2015. She has around five years research experience and published three papers in SCI journals and presented four papers in various national/international conferences.

Vinod Patidar is working as a Professor and Head of Physics at Sir Padampat Singhania University, Udaipur, India. He has obtained his $\mathrm{PhD}$ degree in the field of nonlinear dynamics in 2005 from MLS University, Udaipur, India. He has published more than 55 research papers in various refereed international/national journals/proceedings which have received more than 1,800 citations. He has been a Visiting Guest Scientist at the HISP, University of Potsdam, Germany and ICTP Trieste, Italy in 2003 and 2005 respectively. $\mathrm{He}$ has also presented research papers at various international conferences held in Italy, Germany, Greece, Canada, Nepal, UK and South Korea.

\section{Introduction}

The study of effect of nonlinearity on the behaviour of natural and man-made devices has been an active area of research during last few decades. Particularly the study of chaos (its existence and analysis) in complex dynamical systems has been of interest for scientists, engineers and physicists (Sparrow, 1982; Rossler, 1976). There has been recent interest to study the nonlinear phenomena and chaos in various physical systems through modelling them via electronic circuits (Chua and Lin, 1990; Carroll and Pecora, 1991). For simulating the real complex nonlinear systems, electronic circuits have attracted special attention of researchers in the field due to the fact that electronic circuits provide a very useful medium for both modelling and experimental studies of nonlinear systems. And moreover the real complex physical system can be simulated in real-time through an electronic circuit costing very less (see Murali et al., 1994; Kurt et al., 2000; Kasap and Kurt, 1998; Kiers et al., 2004). Many of these circuits could be modelled using second-order nonlinear differential equations. A simple circuit consisting of a resistor, inductor and diode showed the period doubling route to chaos (Linsay, 1981). Number of circuits such as Matsumoto's, Chua's, Bronson's and mixed mode have been explored (see Murali et al., 1994; Matsumoto et al., 1987; Bitton et al., 1997) and found to exhibit very rich nonlinear behaviours including periodic, quasiperiodic, quasi-periodic and folded-torus, chaotic etc. (see Kiers et al., 2004; Ikezi et al., 1983; Su et al., 1989; Murali et al., 1995). Matsumoto et al. (1987) reported a torus breakdown in the three segment piece-wise sub-circuit. A dissipative non-autonomous chaotic circuit was proposed by 
Murali et al. (1994) using a complex simulation, where the Chua's diode was considered as the source for nonlinearity. Some physical mechanisms have been reported in a simple nonlinear circuit (Bitton et al., 1997) based on the proposal of Matsumoto (1984). Vincent et al. (2014) suggested that the chaotic behaviour could be conveniently tamed in practical experiment. Various nonlinear electronic circuits have been designed and analysed to understand the dynamics of many complex physical systems of diverse nature under real-time conditions like: nonlinear physical pendulum, forced Duffing oscillator, forced van der pol oscillator, forced Duffing-van der pol oscillator, parametrically driven Duffing oscillator etc. (Murali and Lakshmanan, 1996).

Recently, Sharma et al. (2012) have analysed the dynamical behaviour of forced Duffing oscillator under the presence of nonlinear damping term analytically as well as computationally. They have particularly observed the effect of nonlinear damping on the global dynamical behaviour of forced Duffing oscillator through Melnikov analysis, characterisation of parameter space through Lyapunov spectrum calculation, fractalness of basin boundaries and fractal dimensions etc. In this communication we intend to study the same dynamical system i.e., the forced Duffing oscillator system under the presence of nonlinear damping experimentally through an analogue circuit. For this purpose we have designed a circuit and observed its dynamical behaviour under various sets of control parameters and also compared the experimental results with the numerical simulation results. In the next paragraph we briefly describe the forced and quadratically damped Duffing oscillator system.

Duffing oscillator describes the motion of a particle in double-well potential and it is represented by a ubiquitous nonlinear differential equation (Sharma et al., 2012). It has a number of applications not only in physical and engineering problems but also in biological problems. The generalised form of nonlinear equation of Duffing oscillator is:

$$
\ddot{x}+\alpha \ddot{x}-\omega_{o}^{2} x+\beta x^{3}=f \cos \omega t
$$

where first term represents the force on a unit mass particle, second term is velocity term in which $\alpha$ is damping coefficient, whose value is greater than zero. Third term represents the restoring force in which $\omega_{o}$ is natural frequency. Fourth term is nonlinear term in which nonlinear parameter $\beta$ is known as stiffness constant. The right hand side term represents the external force applied to the system which is cosine in nature and $\mathrm{f}$ and $\omega$ are the external forcing amplitude and external frequency respectively. In our present work we consider a nonlinear damping term i.e., proportional to the $p^{\text {th }}$ power of velocity. Thus we consider the following generalised form of model equation:

$$
\ddot{x}+\alpha \dot{x}|\dot{x}|^{p-1}-\omega_{o}^{2} x+\beta x^{3}=f \cos \omega t
$$

Here modulus of velocity is taken to consider the sign of velocity. For all calculations we have considered $\omega_{o}^{2}=1, \beta=1$ and $\omega=1$. We have studied the dynamics of the system for different value of damping coefficient $(\alpha)$ and external forcing amplitude (F). For our study, we have designed an electronic circuit for the model equation (2) and simulated it through the PSpice circuit simulator and implemented it in terms of a real-time circuit for the laboratory simulations. In the next section we give the description of the electronic circuit designed for our study. In Section 3, we discuss our circuit simulation results and compare them with the PSpice simulations as well as numerical simulation results and Section 4 concludes the paper. 


\section{Description of the circuit}

We have constructed an analogue circuit equivalent to equation (2) using conventional operational amplifiers and four quadrant multipliers, which is shown in Figure 1.

The circuit is constructed using conventional operational amplifiers and four quadrant multipliers. Here A1 and A2 represent two integrators; A3, A4 and A5 are inverters; A6 is an absolute rectifier; M1, M2, M3 and M4 are four quadrant multipliers; and A7 is a summing amplifier. The operational amplifiers used in A1, A2, A3, A4, A5, A6 and A7 are op-amp $\mu \mathrm{A} 741 \mathrm{C}$ and the four multipliers M1, M2, M3 and M4 are analogue devices multiplier AD633 with the inbuilt gain of 0.1. Analogue devices multiplier AD633 is the low cost chip with $2 \%$ error at full scale.

Figure 1 Analogue circuit for the forced and quadratically damped Duffing oscillator, equivalent to equation (2) (see online version for colours)

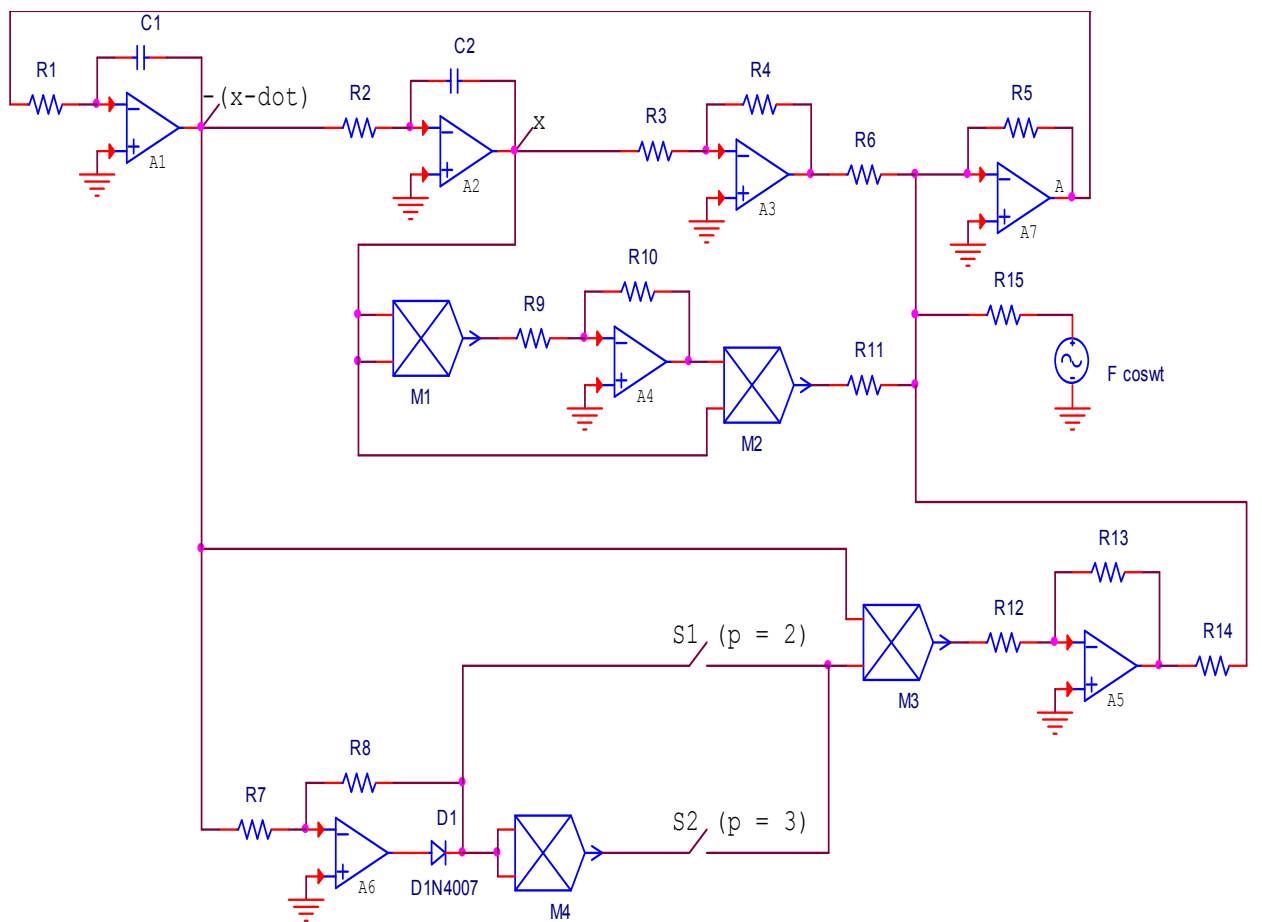

At junction A i.e., at the output of summing amplifier, we obtain the following equation:

$$
\ddot{x}=-\alpha \dot{x}|\dot{x}|^{p-1}+\omega_{0}^{2} x-\beta x^{3}-F \cos \omega t
$$

where $p=2$ (switch) S1 closed) or 3 (switch S2 closed), $\left|\omega_{0}^{2}\right|=\frac{R 5}{R 6}, \alpha=\frac{R 5}{R 14}$, and $\beta=\frac{(0.1) R 5}{R 11}$. 
The values of various resistors and capacitors used in the circuit are as given below:

$$
\begin{aligned}
& R 1=100 \mathrm{k} \Omega, R 2=100 \mathrm{k} \Omega, R 3=1 \mathrm{k} \Omega, R 4=1 \mathrm{k} \Omega, R 5=10 \mathrm{k} \Omega, R 6=10 \mathrm{k} \Omega, R 7=1 \mathrm{k} \Omega, \\
& R 8=1 \mathrm{k} \Omega, R 9=1 \mathrm{k} \Omega, R 10=10 \mathrm{k} \Omega, R 11=1 \mathrm{k} \Omega, R 12=1 \mathrm{k} \Omega, R 13=10 \mathrm{k} \Omega(\text { for } p=2) \\
& \text { or } 100 \mathrm{k} \Omega(\text { for } p=3), R 14=20 \mathrm{k} \Omega, R 15=10 \mathrm{k} \Omega, \text { and } C 1=C 2=0.01 \mu F .
\end{aligned}
$$

Here $\omega_{0}^{2}=1, \alpha=0.5, \beta=1$ and $\omega=1 . \omega=1$ is obtained at external frequency $\mathrm{f} \approx 160 \mathrm{~Hz}$ since here $\omega=2 \pi * f *(R 1 * C 1)$ or $2 \pi * f^{*}(R 2 * C 2)$.

A snapshot of the real circuit implemented on the breadboard with the simulation result on digital CRO for the selected set of parameters $\left(\omega_{0}^{2}=1, \alpha=0.5, \beta=1, \omega=1\right.$, $p=2$ and forced amplitude $\mathrm{F}=0.845 \mathrm{~V}$ ) has been depicted in Figure 2 .

Figure 2 A snapshot of the real circuit (see online version for colours)

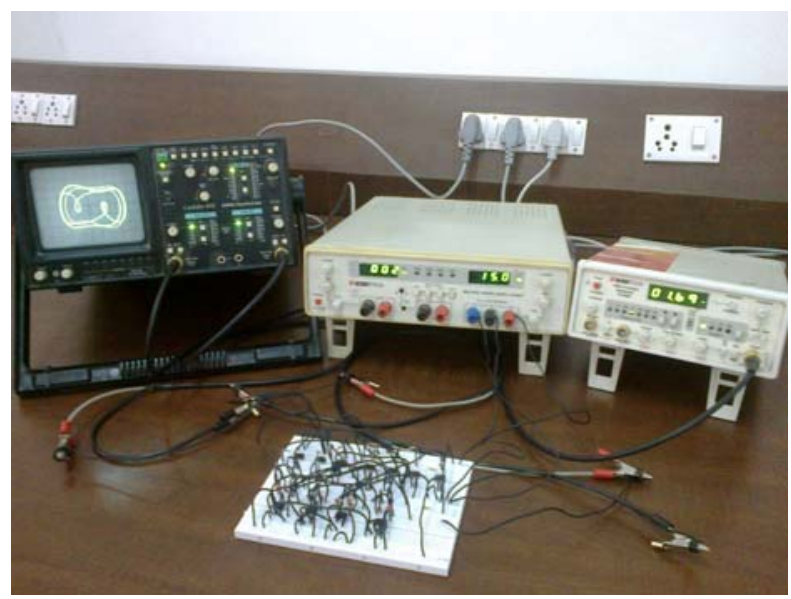

\section{Results and discussion}

In this section we present the results of experimental study and their comparison with the numerical results. In Figure 3, we show the result for the fixed values of parameters $\omega_{0}^{2}=1, \beta=1, \omega=1, \alpha=0.5, p=2$ (drag like damping) and various values of forcing amplitudes. In Figure 3(a), we have depicted a numerically computed bifurcation diagram showing the complete sequence of dynamical behaviour for the selected set of parameters against the various values of forcing amplitude. However in Figure 3(b), the first column depicts the value of the forcing amplitude and the corresponding numerical simulation results produced by integrating the second order differential equation (2) through MATLAB, the second and third columns respectively show thePSpice simulator results and experimental results along with the corresponding values of forcing amplitudes. 
Figure 3 (a) Bifurcation diagram for the nonlinearly damped forced Duffing oscillator for $\omega_{0}^{2}=1, \beta=1, \omega=1, \alpha=0.5, p=2$ (drag like damping) (b) Phase plots for the nonlinearly damped forced Duffing oscillator (see online version for colours)

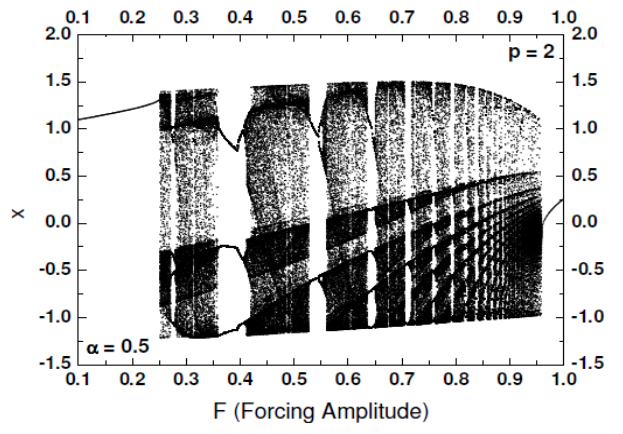

(a)

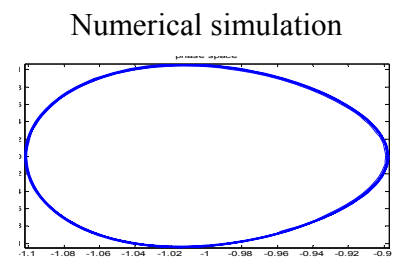

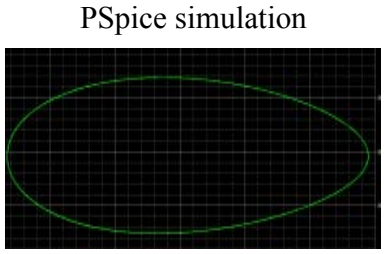

$\mathrm{F}=0.1$

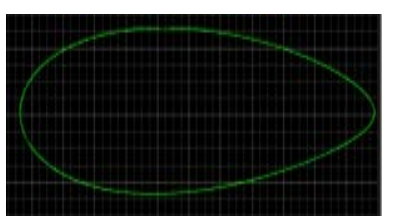

$\mathrm{F}=0.2$

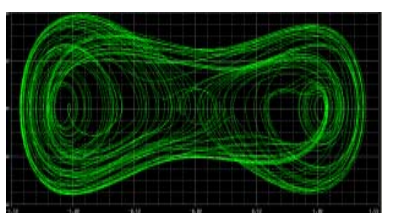

$\mathrm{F}=0.28$

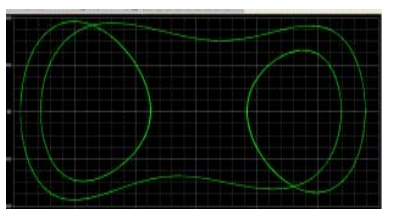

$\mathrm{F}=0.37$

(b)

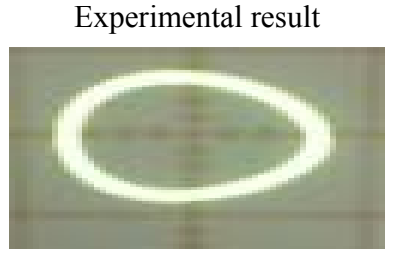

$\mathrm{F}=0.1$

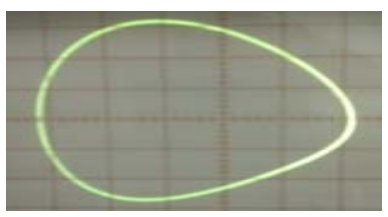

$\mathrm{F}=0.2$

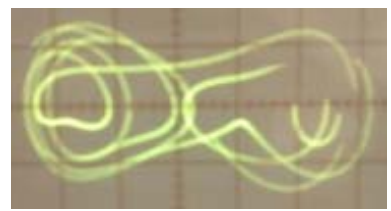

$\mathrm{F}=0.425$

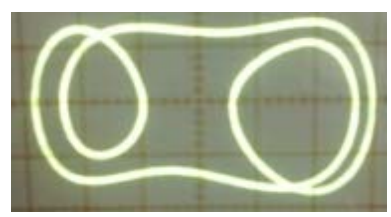

$\mathrm{F}=0.52$

Notes: First, second and third columns in Figure 3(b) respectively show the computationally obtained phase plots, PSpice simulation results and the third the experimental results obtained from the circuit shown in Figure 1. 
Figure 3 (a) Bifurcation diagram for the nonlinearly damped forced Duffing oscillator for $\omega_{0}^{2}=1, \beta=1, \omega=1, \alpha=0.5, p=2$ (drag like damping) (b) Phase plots for the nonlinearly damped forced Duffing oscillator (continued) (see online version for colours)

Numerical simulation

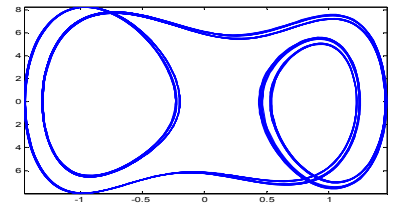

$\mathrm{F}=0.407$

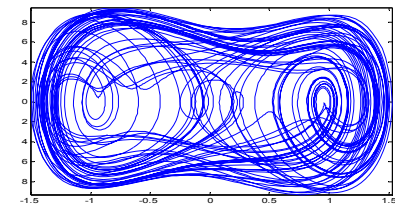

$\mathrm{F}=0.5$

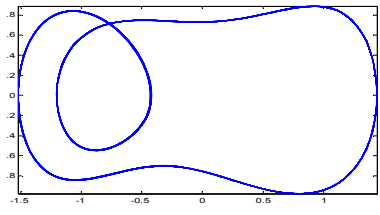

$\mathrm{F}=0.538$

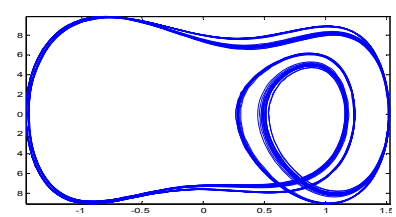

$\mathrm{F}=0.55$

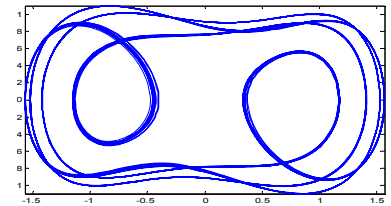

$\mathrm{F}=0.645$

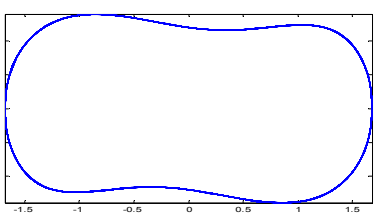

$\mathrm{F}=1$
PSpice simulation

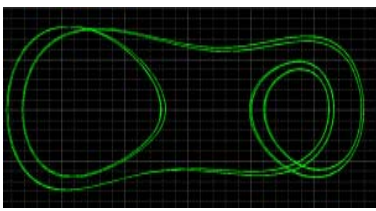

$F=0.395$

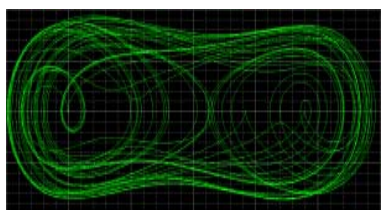

$\mathrm{F}=0.48$

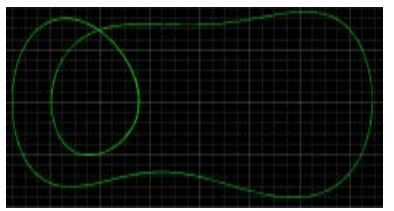

$F=0.52$

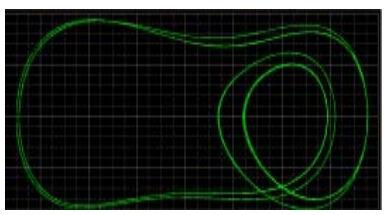

$\mathrm{F}=0.54$

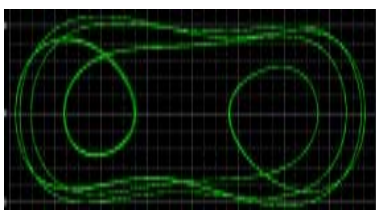

$F=0.637$

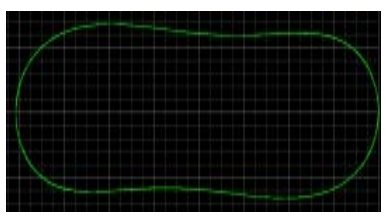

$\mathrm{F}=0.95$

$F=0.95$

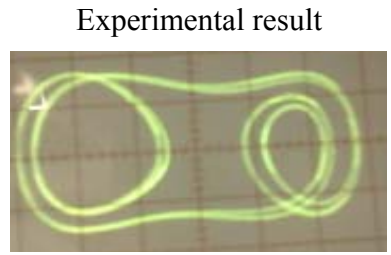

$\mathrm{F}=0.53$

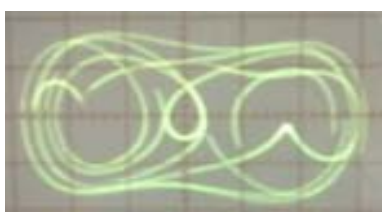

$F=0.62$

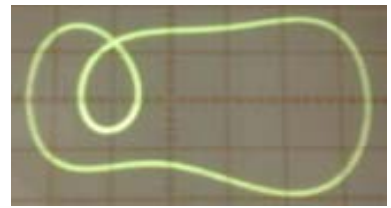

$F=0.67$

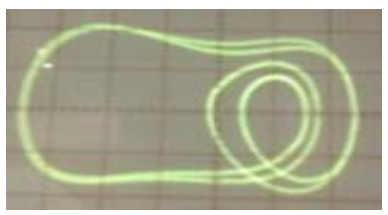

$F=0.73$

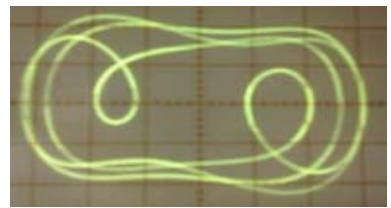

$\mathrm{F}=0.845$

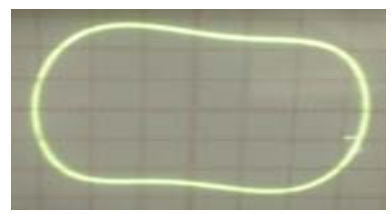

$F=1.29$

(b)

Notes: First, second and third columns in Figure 3(b) respectively show the computationally obtained phase plots, PSpice simulation results and the third the experimental results obtained from the circuit shown in Figure 1. 
Figure 4 (a) Bifurcation diagram for the nonlinearly damped forced Duffing oscillator for $\omega_{0}^{2}=1, \beta=1, \omega=1, \alpha=0.5, p=3$ (nonlinear third-power damping) (b) Phase plots for the nonlinearly damped forced Duffing oscillator (see online version for colours)

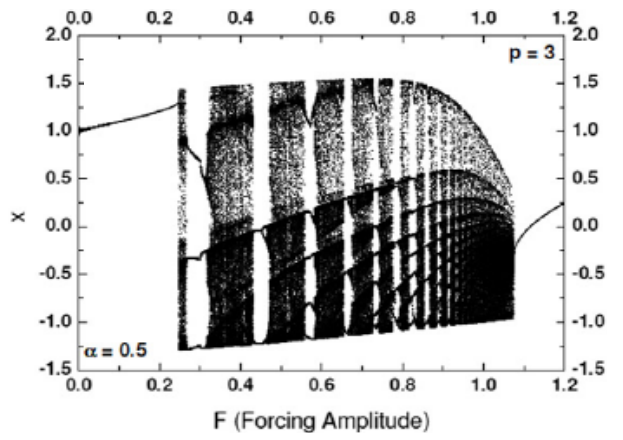

(a)

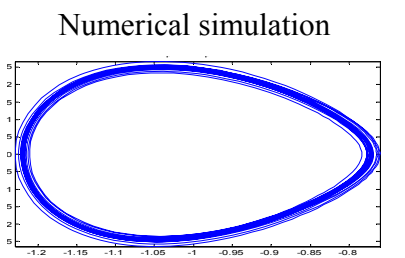

$\mathrm{F}=0.2$

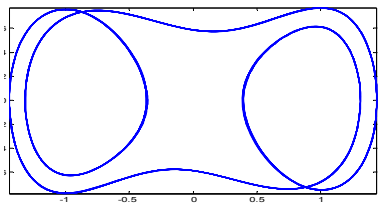

$\mathrm{F}=0.3$

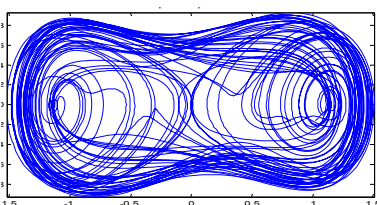

$\mathrm{F}=0.4$

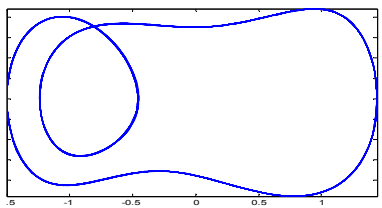

$\mathrm{F}=0.45$
PSpice simulation

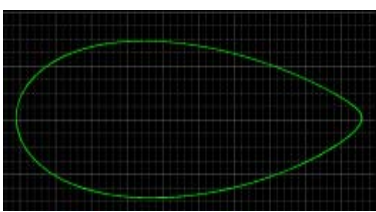

$\mathrm{F}=0.32$

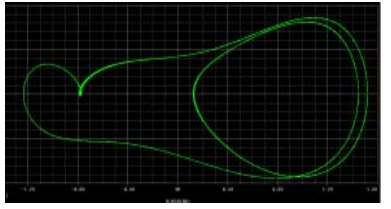

$\mathrm{F}=0.35$

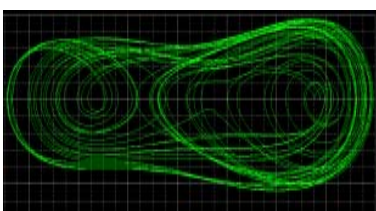

$\mathrm{F}=0.48$

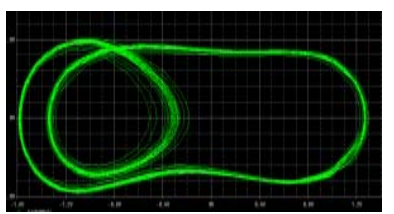

$\mathrm{F}=0.55$
Experimental result

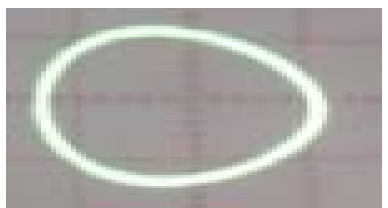

$\mathrm{F}=0.45$

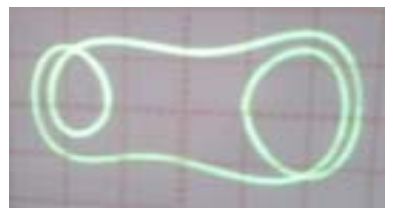

$\mathrm{F}=0.73$

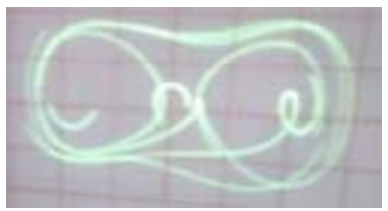

$\mathrm{F}=0.98$

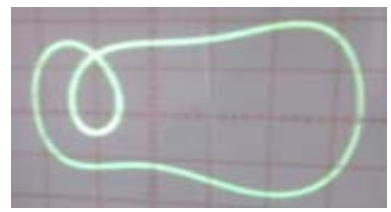

$\mathrm{F}=1.15$

(b)

Notes: First, second and third columns in Figure 4(b) respectively show the computationally obtained phase plots, PSpice simulation results and the third the experimental results obtained from the circuit shown in Figure 1. 
We observe from Figure 3 that the designed circuit is able to produce the correct sequence of the dynamical behaviour as obtained with the numerical simulation however the corresponding values of the forcing amplitudes are shifted to lower side for the PSpice simulations and to the upper side for the experimental simulations. This difference in the values of forcing amplitude can be explained in the following way: in PSpice, the difference in the forcing amplitude is due to the input and output offset voltages of analogue devices multiplier IC AD633 that creates 2\% error at full scale however in the experimental circuit, the difference in forcing amplitude is due to AD633 and $\pm 10-15 \%$ tolerances of electronic components used.

Another set of results for the fixed values of parameters $\omega_{0}^{2}=1, \beta=1, \omega=1, \alpha=0.5$, $p=3$ (nonlinear third-power damping) and various values of forcing amplitudes have been depicted in Figure 4. Particularly in Figure 4(a), we have depicted a numerically computed bifurcation diagram showing the complete sequence of dynamical behaviour for the selected set of parameters against the various values of forcing amplitude. However in Figure 4(b), the first column depicts the value of the forcing amplitude and the corresponding numerical simulation results produced by integrating the second order differential equation (2) through MATLAB, the second and third columns respectively show the PSpice simulator results and experimental results along with the corresponding values of forcing amplitudes. We observe that the difference in the forcing amplitude values for the numerical computation, Pspice simulation and experimental results is large as compared to the case $p=2$ (Figure 3) is due to use of one additional analogue multiplier IC AD633.

\section{Conclusions}

We have designed and implemented an analogue circuit equivalent to a forced and quadratically damped Duffing oscillator to study the effect of nonlinear damping on the dynamical behaviour of the forced oscillator. We have studied the designed circuit extensively for various combinations of system parameters and observed that the designed circuit is able to produce the correct sequence of the dynamical behaviour as obtained with the numerical simulation. We have also compared the experimental results, PSpice simulations and numerical results and found good degree of agreement. The designed circuit may be useful to understand the real time behaviour of complex physical systems modelled by forced and quadratically damped Duffing differential equation.

\section{Acknowledgements}

We acknowledge the research facility provided by Sir Padampat Singhania University in terms of required software as well as the laboratory facility to implement the experimental circuit of forced Duffing oscillator 


\section{References}

Bitton, G., Balberg, I., Gomez, M. and Weisz, S.Z. (1997) 'Numerical and analytical study of nonlinear electronic circuits', Int J. Bifurcation Chaos, Vol. 7, No. 5, pp.1065-1074.

Carroll, T.L. and Pecora, L.M. (1991) 'Synchronizing chaotic circuits', IEEE Trans Circ Syst, Vol. 38, No. 4, pp.453-456.

Chua, L.O. and Lin, G.N. (1990) 'Canonical realization of Chua's circuit family', IEEE Trans Circ Syst, Vol. 37, No. 7, pp.885-902.

Ikezi, H., de Grassie, J.S. and Jensen, T. (1983) 'Observation of multiple-valued attractors and crises in a driven nonlinear circuit', Phys Rev A, Vol. 28, No. 2, pp.1207-1209.

Kasap, R. and Kurt, E. (1998) 'Investigation of chaos in the RL-diode circuit by using the BDS test', J Appl Math Decis Sci, Vol. 2, No. 2, pp.193-199.

Kiers, K., Schmidt, D. and Sprott, J.C. (2004) 'Precision measurements of a simple chaotic circuit', Am J Phys, Vol. 72, No. 4, pp.503-509.

Kurt, E., Acar, S. and Kasap, R. (2000) 'A comparison of chaotic circuits a statistical approach', Math Comput Appl J, Vol. 5, No. 2, pp.95-104.

Linsay, P.S. (1981) 'Period doubling and chaotic behavior in a driven anharmonic oscillator', Phys Rev Lett, Vol. 47, No. 19, pp.1349-1352.

Matsumoto, T. (1984) 'A chaotic attractor from Chua's circuit', IEEE Trans Circ Syst, Vol. CAS 31, No. 12, pp.1055-1058.

Matsumoto, T., Chua, L.O. and Tokunaga, R. (1987) 'Chaos via torus breakdown', IEEE Trans Circ Syst, Vol. 34, No. 3, pp.240-253.

Murali, K. and Lakshmanan, M. (1996) Chaos in Nonlinear Oscillators: Controlling and Synchronization, World Scientific Publishing Co. Pte Ltd., Singapore.

Murali, K., Lakshmanan, M. and Chua, L.O. (1994) 'The simplest dissipative nonautonomous chaotic circuit', IEEE Trans Circ Syst, Vol. 41, No. 6, pp.462-463.

Murali, K., Lakshmanan, M. and Chua, L.O. (1995) 'Controlling and synchronization of chaos in the simplest dissipative non-autonomous circuit', Int. J. Bifurcation Chaos, Vol. 5, No. 2, pp.563-571.

Rossler, O.E. (1976) 'An equation for continuous chaos', Phys Lett A, Vol. 57, No. 5, pp.397-398.

Sharma, A., Patidar, V., Purohit, G. and Sud, K.K. (2012) 'Effects on the bifurcation and chaos in forced Duffing oscillator due to nonlinear damping', Commun Nonlinear Sci Numer Simulat, Vol. 17, No. 6, pp.2254-2269.

Sparrow, C. (1982) The Lorentz Equations: Bifurcation, Chaos and Strange Attractor, Springer, New York.

$\mathrm{Su}, \mathrm{Z}$., Rollius, R. and Hunt, J.R. (1989) 'Universal properties at the onset of chaos in driven diode resonator systems', Phys Rev A, Vol. 40, No. 5, pp.2689-2697.

Vincent, U.E., Nana Nbendjo, B.R., Ajayi, A.A., Njah, A.N. and McClintock, P.V.E. (2014) 'Hyperchaos and bifurcations in a driven Van der Pol Duffing oscillator circuit', International Journal of Dynamics and Control, Vol. 3, No. 4, pp.363-370. 